\title{
Fabrication of suspended metallic structures: application to a one-shot micro-valve
}

\author{
A. Debray ${ }^{1 \mathrm{a})}$, K. Ueda ${ }^{1}$, M. Shibata ${ }^{1}$, and H. Fujita ${ }^{2}$ \\ ${ }^{1}$ Canon Research Center, Canon Inc. \\ 3-30-2 Shimomaruko, Ohta-ku, Tokyo 146-8501, Japan \\ ${ }^{2}$ Institute of Industrial Science, University of Tokyo, \\ 4-6-1 Komaba, Meguro-ku, Tokyo 153-8505, Japan \\ a)debray.alexis@canon.co.jp
}

Abstract: We developed a method which allows us to fabricate suspended metallic structures without the need for a sacrificial layer. It consists in the selective dip-coating of a low melting point alloy on etched silicon patterns. The technique was applied to the fabrication of a passive one-shot micro-valve sensitive to the ambient temperature and the input pressure. The working principle of the valve was validated. The primary advantage of the system is its low opening pressure difference (a few to tens of kilopascal).

Keywords: surface tension, fuel cell, MEMS, one-shot micro-valve, suspended metallic structures

Classification: Micro- or nano-electromechanical systems

\section{References}

[1] C. L. Chua, D. K. Fork, and K. Van Schuylenbergh, "Out-of-plane high-Q inductors on low-resistance silicon," J. Microelectromech. Syst., vol. 12, no. 6, pp. 989-995, 2003.

[2] J. B. Yoon, B. I. Kim, Y. S. Choi, and E. Yoon, "3-D construction of monolithic passive components for RF and microwave ICs using thickmetal surface micromachining technology," IEEE Trans. Microw. Theory Tech., vol. 51, no. 1, pp. 279-288, 2003.

[3] A. Debray, M. Shibata, and H. Fujita, "Low melting point alloy as a functional material for a one-shot micro-valve," J. Micromech. Microeng, vol. 17, no. 8, pp. 1442-1450, 2007.

[4] J. Bejhed, P. Rangsten, and J. Köhler, "Demonstration of a single use microsystem valve for high gas pressure applications," J. Micromech. Microeng, vol. 17, no. 3, pp. 472-481, 2007.

[5] D. H. Gracias, J. Tien, T. L. Breen, C. Hsu, and G. M. Whitesides, "Forming electrical networks in three dimensions by self-assembly," Science, vol. 289, pp. 1170-1172, 2000. 


\section{Introduction}

The fabrication of suspended metallic micro-structures usually includes the etching of a sacrificial material $[1,2]$. Here, we present a simple process taking advantage of surface tensions to produce suspended metallic microstructures by directly dipping the sample into a melted alloy. The easiness of the fabrication is balanced by the limitation on the obtained shapes which are given by the minimization of surface energies. Another advantage of this process is the good adhesion between the alloy and the substrate and the possibility to obtain closed contours.

This process was used to fabricate a one-shot micro-valve. It consists of a silicon wafer perforated through its thickness by a channel which is obstructed on one side by a low melting point alloy. The device is depicted in figure 1 . When the ambient temperature is below the melting temperature of the alloy $T_{m}$, this last is solid and presents a high mechanical strength to pressure differences across the channel (figure 1-a). When the ambient temperature is above $T_{m}$, the alloy becomes liquid and its mechanical strength is determined by its surface tension. It can be broken by applying an appropriate pressure difference across the channel (figure 1-b).

One-shot micro-valves find application in chemistry to trigger chemical reactions, biology for sampling, micro fuel cells for safety purpose and assembly, and in micro-rockets for propulsion. Several one-shot micro-valves have been reported (see [3] for a short review). They usually include materials such that the operating temperature cannot be tuned easily. This limitation was recently alleviated by the use of a low melting point alloy which melting temperature can be easily tuned by changing its composition [3]. However, this was only possible by incorporating a metallic multilayer between the channel and the alloy, resulting in opening pressure difference higher than $50 \mathrm{kPa}$. Moreover, the experimental study revealed a mechanical interaction between the multilayer and the alloy, the design of the opening pressure difference being difficult below $100 \mathrm{kPa}$. Separately Bejhed et al recently re-
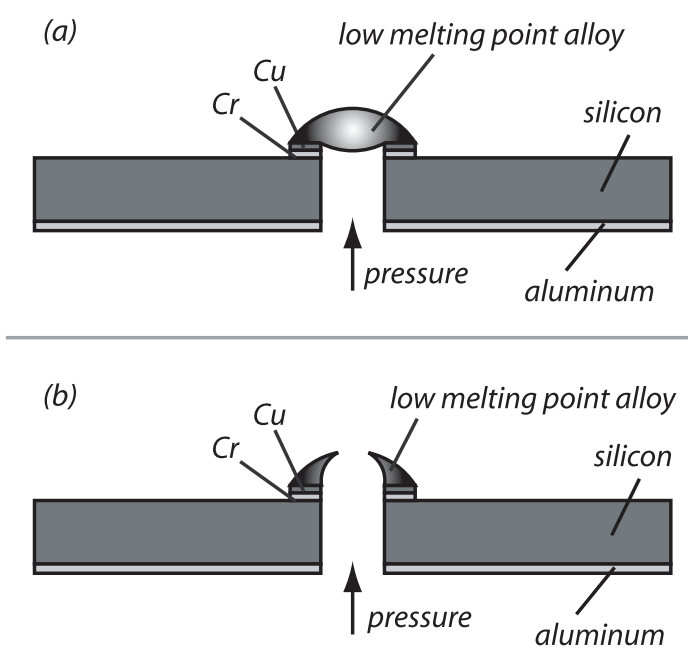

Fig. 1. Scheme of the cross-section of the device: (a) in the closed position; (b) in the open position. 
ported a one-shot micro-valve using a low melting point alloy and made by screen-printing [4]. The opening pressure difference was above $50 \mathrm{kPa}$ and not controlled. Moreover the fabrication process was complex. Our new fabrication method allows lower and controllable opening pressure differences using a simple process.

The paper first shows the fabrication process of the device and the experimental conditions. The results are then presented and discussed. The article focuses on the results of the fabrication and the demonstration of the working principle.

\section{Method}

\subsection{Fabrication}

The fabrication started with a double-side polished silicon wafer. Using a lift-off process (ZPN 1150-90 photoresist), chromium and copper layers were successively deposited by sputter and then patterned on the front side of the wafer. Aluminum was then thermally evaporated and patterned by photolithography (Shipley S1805 photoresist) and metal etching on the back side of the wafer. Using the aluminum layer as a mask, silicon was etched from the back side by deep RIE (Reactive Ion Etching). The wafer was then manually dipped into a two-phase solution at $60^{\circ} \mathrm{C}$ containing on the top $\mathrm{HCl}$ diluted in water ( $\mathrm{pH} 1)$ and on the bottom the melted alloy (LMA-117, Small Parts Inc; melting temperature: $47^{\circ} \mathrm{C}$ ) [5]. Depending on the sample, the channel was either 100 or $200 \mu \mathrm{m}$ in diameter and the width of the alloy part was $300,400,500$ or $600 \mu \mathrm{m}$.

\subsection{Characterization of the device}

Three characteristics were measured: the leakage rate and the maximum applicable pressure difference when the alloy was in the solid state, and the opening pressure when the alloy was in the liquid state.

The leakage rate was measured using the $V \Delta P$ method which consists in monitoring the pressure decay of a chamber of constant volume filled with gas and connected to the device. The ambient temperature was $25^{\circ} \mathrm{C}$. The pressure difference was $200 \mathrm{kPa}$ and the gas was hydrogen.

The maximum applicable pressure difference was measured by connecting the input of the device in series with a pressure gage, a manual pump and a water tank. The pressure of the water connected with the device was increased up to $3 \mathrm{MPa}$ during $5 \mathrm{~min}$ at an ambient temperature of $25^{\circ} \mathrm{C}$.

To measure the opening pressure when the alloy was in the liquid state, the device was placed inside a chamber with controlled temperature and relative humidity (SH-641, Espec corp.) and its input was connected to a nitrogen line in series with a pressure sensor (PT-103B-A, Cosmo Instruments), a mass flow meter (SEF-4400R, Horiba STEC) and a pressure regulator (UR7340RC, Horiba STEC). The temperature of the chamber was stabilized at $60^{\circ} \mathrm{C}$ and the nitrogen pressure was raised at a speed of $1.5 \mathrm{kPa} \mathrm{s}^{-1}$ until a large flow rate was recorded. 

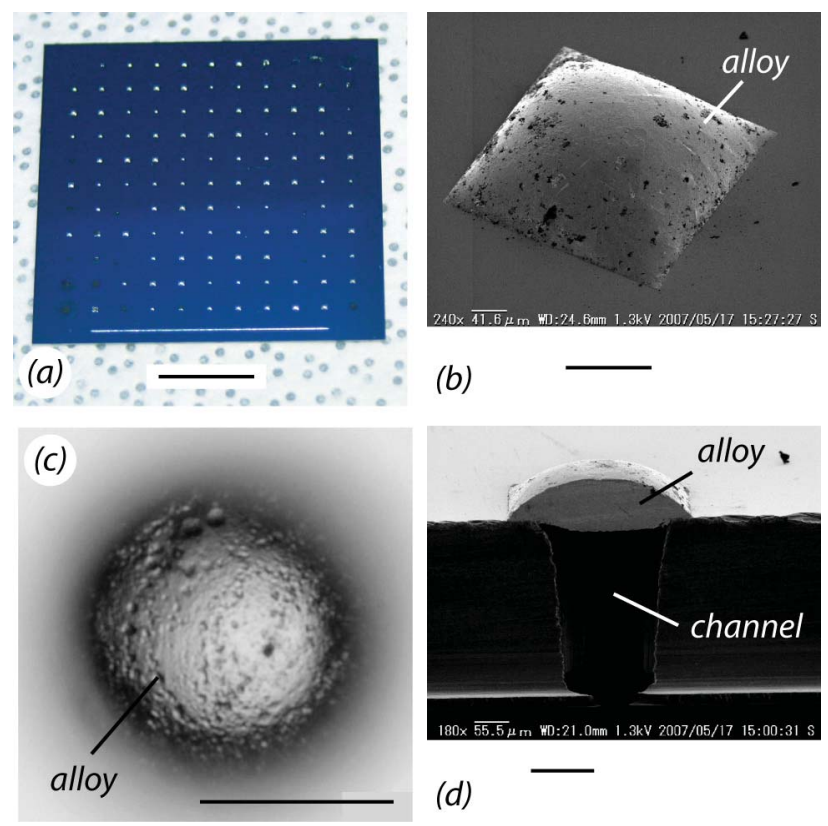

Fig. 2. Results from the fabrication. (a) A full wafer after fabrication; bar length $10 \mathrm{~mm}$. (b) SEM micrograph of a device; bar length $100 \mu \mathrm{m}$. (c) Optical micrograph of the alloy part seen from the channel; bar length $100 \mu \mathrm{m}$. (d) SEM micrograph of the cross section of a device; bar length $100 \mu \mathrm{m}$.

\section{Results and Discussion}

\subsection{Fabrication}

Figure 2-a shows a photography of the front side of a full wafer after fabrication. The white dots are alloy parts. The alloy coated only the copper pads and not the silicon nor the aluminum. The successful fabrication yield for this wafer was $84.6 \%$ with no correlation to the size of the device. The failed samples were not covered by the alloy because of photoresist remaining on the copper pads from the silicon process. Figure 2-b shows a SEM micrograph of the alloy part of one sample. The alloy part is clearly defined by the copper pad. Some impurities coming from the melted alloy bath are seen on the alloy part. Figure 2-c shows an optical micrograph of the backside of one device. We can see the alloy surface on the back side being regular and that the alloy did not penetrate inside the channel. This last asssertion is confirmed by figure 2-d which shows the SEM micrograph of the cross-section of one sample obtained by dicing saw. The alloy part is clearly seen suspended over the silicon channel.

The reasons for the selective dip-coating can be explained as follows. The diluted $\mathrm{HCl}$ etched the copper oxide naturally present at the surface of the copper pad. Because the energy of the water-copper interface is higher than that of the alloy-copper interface, the alloy coated the copper layer. On the other hand, the interfacial energy of water with the aluminum and silicon oxides is smaller than that of the interface of the alloy with these materials and therefore, the alloy did not coat them. When the sample was removed 
from the bath, the alloy did not wet the silicon dioxide because its critical surface tension $\left(0.55 \mathrm{~N} \mathrm{~m}^{-1}\right)$ is higher than the critical surface tension of silicon dioxide $\left(0.037 \mathrm{~N} \mathrm{~m}^{-1}\right)$. During the deep-RIE process, the interior of the channel was coated by a fluorocarbon layer (critical surface tension of $0.018 \mathrm{~N} \mathrm{~m}^{-1}$ ). Therefore, the melted alloy and the diluted $\mathrm{HCl}$ (the surface tension of water being $0.072 \mathrm{~N} \mathrm{~m}^{-1}$ ) did not coat it.

Figure 2-d shows the two alloy-air interfaces, one in the channel and one above the silicon wafer. For liquid-gas interfaces, the Laplace equation stipulates that $\Delta P=2 \gamma / R$, with $\Delta P$ the pressure difference between the liquid and the gas, $\gamma$ the surface tension of the liquid and $R$ the radius of curvature of the liquid-gas interface. If the alloy solidifies in air, then, according to this equation, the radius of curvature of both interfaces should be the same. Measurement of these radius of curvature using the sample shown in figure 2-d yielded a radius of $160.0 \mu \mathrm{m}$ for the upper interface and $165.0 \mu \mathrm{m}$ for the lower one, which are similar.

The melted alloy structure remains suspended thanks to the tension of the alloy-air interface. The Tate law states that the maximum weight of a pendant drop which can be supported on a circular border is $2 \pi R_{c} \gamma$, with $R_{c}$ the radius of the border and $\gamma$ the surface tension of the liquid constituting the drop. In our case, this is only a first approximation as part of the weight of the alloy is supported by the upper surface of the silicon wafer. For example, the weight of a $200 \mu \mathrm{m}$ diameter alloy sphere generates a downward force of $3.8 \times 10^{-7} \mathrm{~N}$ (density of alloy $9160 \mathrm{~kg} \mathrm{~m}^{-3}$ ), while the surface tension of a circular border $100 \mu \mathrm{m}$ in radius can generate a maximum upper force of $3.5 \times 10^{-4} \mathrm{~N}$. This simple calculation allows to think that larger suspended structures are possible.

\subsection{Working principle}

The leakage rate with the alloy in the solid state was measured to be $2.3 \times$ $10^{-10} \mathrm{~mol} \mathrm{~s}^{-1}$ using hydrogen at a pressure difference of $200 \mathrm{kPa}$. This is very low and indicates a good sealing between the alloy and the copper layer. This leakage rate corresponds to a $10 \%$ energy loss during years for micro fuel cells, whereas the energy loss for lithium batteries is $10 \% /$ month. This demonstrates the utility of this device as a primary connector for micro fuel cells.

The maximum supported pressure difference with the solid alloy was more than $3 \mathrm{MPa}$ for a width of alloy of $400 \mu \mathrm{m}$ and a channel diameter of $200 \mu \mathrm{m}$. When the alloy was melted, the opening pressure difference was in the order of a few kilopascal. For example, two valves with an alloy width of $500 \mu \mathrm{m}$ opened at a pressure difference of $18.0 \mathrm{kPa}$ and $10.1 \mathrm{kPa}$ for a channel radius of $100 \mu \mathrm{m}$ and $200 \mu \mathrm{m}$ respectively. A resistance to high pressure differences when the alloy is solid and a relatively low opening pressure when the alloy is melted demonstrate the working principle of the system.

As the pressure in the channel is increased, the alloy-air interface in the channel deforms and lifts up until reaching the upper alloy-air interface. Figure 3-a shows an SEM micrograph of a valve being opened. A hole was 


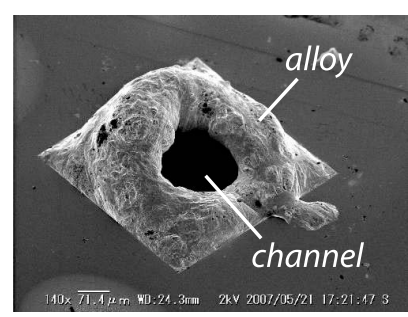

(a)

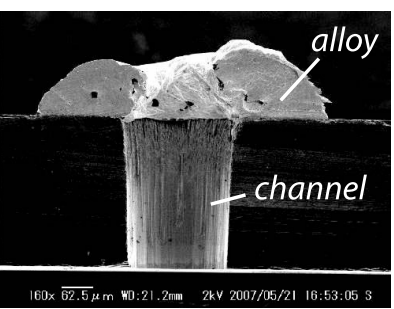

(b)

Fig. 3. Opened valves; (a) SEM micrograph of an opened device; bar length $100 \mu \mathrm{m}$. (b) SEM micrograph of the cross section of an opened device; bar length $100 \mu \mathrm{m}$.

created in the alloy above the channel and much of the alloy seems to remain. Figure 3-b shows the SEM micrograph of the cross section of a valve being opened. It is interesting to note that the melted alloy was anchored at the intersection of the copper pad and the channel insuring a good control of the opening pressure. Moreover we can note that the lower alloy-air interface was developed in a regular, probably circular shape.

Finally, the dimensional analysis of the system is presented. The dimension of the pressure $\left(\mathrm{L}^{-1} \mathrm{MT}^{-2}\right)$ being inversely proportional to a length and the dimension of the surface tension $\left(\mathrm{MT}^{-2}\right)$ being independent of the length, the opening pressure difference can be modified by simply scaling the size of the device. For example, two valves, one having all its lengths double than the other will have an opening pressure twice smaller than the second one.

\section{Conclusions}

Suspended metallic micro-structures made by the selective dip-coating of alloy were successfully fabricated. This technique was used to produce a passive one-shot micro-valve sensitive to the ambient temperature and the input pressure. The experimental characterization showed that opening pressures in the order of a few kilopascal can be achieved. The main advantage of this one-shot micro-valve is the low opening pressure difference compared to previous similar systems.

Finally, we believe that this new fabrication method could find applications in other devices, for example in suspended micro-electric wires, which are interesting for insulating them from the substrate (to allow cooling for example) or in new compliant and conductive metallic structures, the Young's modulus of low melting point alloys being around $20 \mathrm{GPa}$.

\section{Acknowledgments}

Photomasks were made using the University of Tokyo VLSI Design and Education Center (VDEC)'s 8-inch EB writer F5112+VD01 donated by ADVANTEST corporation. The layout was designed by Cadence Virtuoso accessible through VDEC's academic program. 